\title{
Caracterización trófica de Orestias (Teleostei: Cyprinodontidae) en el Parque Nacional Lauca
}

\section{Trophic characterization of Orestias (Teleostei: Cyprinodontidae) in the Lauca National Park}

\author{
Claudia J. Guerrero ${ }^{1,3,5}$, Elie Poulin ${ }^{1,4}$, Marco A. Méndez ${ }^{2}$ \& Irma Vila ${ }^{3 *}$ \\ ${ }^{1}$ Laboratorio de Ecología Molecular, Departamento de Ciencias Ecológicas, Facultad de Ciencias, Universidad de Chile, Las \\ Palmeras 3425, Ñuñoa, Santiago. \\ ${ }^{2}$ Laboratorio de Genética y Evolución, Departamento de Ciencias Ecológicas, Facultad de Ciencias, Universidad de Chile, \\ Las Palmeras 3425, Ñuñoa, Santiago. \\ ${ }^{3}$ Laboratorio de Limnología, Departamento de Ciencias Ecológicas, Facultad de Ciencias, Universidad de Chile, Las Palmeras \\ 3425, Ñuñoa, Santiago. \\ ${ }^{4}$ Instituto de Ecología y Biodiversidad de Chile, Las Palmeras 3425, Ñuñoa, Santiago. \\ ${ }^{5}$ Escuela de Medicina Veterinaria sede Talca, Facultad de Recursos Naturales y Medicina Veterinaria, Universidad Santo \\ Tomás. Av. Carlos Schorr 255, Talca, Maule. \\ *limnolog@uchile.cl
}

\section{RESUMEN}

Los peces muestran una amplia diversidad de comportamientos alimentarios, algunas veces asociados con morfologías específicas, las cuales pueden reflejar adaptaciones para usar los recursos dependiendo de su hábitat. En los sistemas acuáticos aislados, los peces son un buen modelo de estudio para entender las relaciones tróficas. Este estudió evaluó la dieta de cinco especies de Orestias, las cuales habitan ocho sitios diferentes en la Región de Arica y Parinacota, Parque Nacional Lauca. Se analizaron 177 estómagos pertenecientes a las especies Orestias chungarensis, O. laucaensis, O. parinacotensis, $O$. piacotensis y Orestias cf agassii. Para los análisis se utilizaron descriptores cuantitativos de número de items presa y frecuencia de ocurrencia. Se analizó la riqueza, diversidad y abundancia de presas por especies y localidad, se evaluó la amplitud y sobrelapamiento de dieta y mediante un análisis de correspondencia (DCA) se analizó la composición dietaria.En general, la dieta de Orestias consistió en zooplancton y macroinvertebrados asociados a las macrófitas como son Cladocera (Chydorus, Daphnia, Alonella), Ostracoda (Cyprinotus). Amphipoda (Hyalella), Mollusca (Biomphalaria) e Insecta (Chironomus). La variación en la riqueza de presas y amplitud de dieta observado entre las poblaciones de Orestias sería el reflejo de una estrategia oportunista de las especies, consumiendo las presas de mayor frecuencia, no observándose diferenciación trófica entre ellas.

Palabras Claves: Altiplano, alopatría, dieta, peces, simpatría.

\begin{abstract}
Fish exhibit a wide diversity of feeding behaviors, sometimes associated with specific morphologies, which may reflect adaptations to exploit the resources depending upon where they live. In isolated aquatic systems, fish provide a good model to study species trophic relationships. The present study evaluates the diet of five species of Orestias which inhabit eight different sites of the Arica and Parinacota Region,Lauca National Park, Chile. We analyzed 177 stomachs of Orestias chungarensis, O.laucaensis, O.parinacotensis, O. piacotensis and Orestias cf agassii. For the analyses we used quantitative descriptors of number of prey items and frequency of occurrence. We analyzed the richness and abundance of prey,diet overlap by species and by location. We analyzed diet composition using an analysis of correspondence (DCA). The diet of Orestias consisted of zooplankton and macroinvertebrates associated with macrophytes such as Cladocera (Chydorus, Daphnia, Alonella), Ostracoda (Cyprinotus). Amphipoda (Hyalella), Mollusca (Biomphalaria) and Insecta (Chironomus). The variation in prey richness and diet amplitude observed among the studied populations of Orestias may reflect an opportunist trophic strategy of these species, consuming the prey species which are most frequent, thus no showing trophic differentiation among them.
\end{abstract}

KeYwords: Altiplano, allopatric, diet, fish, sympatric. 


\section{INTRODUCCIÓN}

El género Orestias es un grupo de peces de interés biogeográfico en Suramérica por su endemismo y adaptación a los sistemas de altura de los Andes. El origen de este género se ha hipotetizado ser Plio-Pleistoceno, con una alta diversificación en los últimos millones de años (Parenti 1984a; Vila et al. 2013).

Actualmente el género presenta 46 especies distribuidas desde el lago Lacsha en el norte del Perú hasta el Salar de Ascotán en el norte de Chile (Arratia 1982; Parenti 1984 a 1984b; Vila \& Pinto 1986; Northcote 2000; Costa 2003; Vila 2006; Vila et al. 2011). De las especies descritas para este género, 15 se encuentran habitando el Lago Titicaca y forman una importante red trófica dentro del mismo ( Maldonado et al 2009). Sin embargo, las especies de Orestias que habitan en el Altiplano sur, se caracterizan por presentar una distribución geográfica restringida a una especie endémica por sistema acuático. Estos sistemas se localizan sobre los $4000 \mathrm{msnm}$, son endorreicos, con diferencias extremas de temperatura diaria y sin interconexiones. Se caracterizan por el desarrollo de un cinturón de macrófitas y fauna asociada similar ( Márquez et al. 2009). Estudios sobre la dieta de Orestias agassii en sistemas como salares,muestra una dieta amplia (Guzman Sepúlveda 2009 ,), sin embargo, estudios sobre el tipo de dieta en la zona más alta del altiplano aún es escasa. En el caso del Parque Nacional Lauca (PNL), los eventos geológicos y volcánicos de la zona que formaron el paisaje y fragmentaron los sistemas acuáticos, habrían separado la población ancestral de Orestias que habitaba el paleorío Lauca en cuatro especies, restringidas la mayoría a un solo sistema (Vila et al. 2013). Para el Lago Chungará se ha descrito Orestias chungarensis Vila \& Pinto 1986, Orestias parinacotensis Arratia 1982 para el bofedal de Parinacota, Orestias piacotensis Vila 2006 en la laguna de Piacota y Orestias laucaensis Arratia 1982 en el río Lauca y Lagunas de Cotacotani. Teniendo en cuenta que los ecosistemas son dinámicos, los individuos que los habitan presentarían variaciones en su dieta, facilitando nuevos "fenotipos" adaptados a las condiciones de los sistemas (Kocher 2004),en el PNL la presencia de una especie y por ende la ausencia de competencia dentro de los sistemas facilitaría dietas generalistas. Bajo esta hipótesis, este estudio intenta caracterizar la trofía de las especies de Orestias del Parque Nacional Lauca.

\section{MATERIALES Y MÉTODOS}

Se capturaron entre las macrófitas, con redes manuales y pesca eléctrica, (permiso de pesca Subsecretaría de Pesca: Resolución 2231, 19 de agosto 2011,Proyecto Fondecyt 1110243), 177 especímenes de Orestias pertenecientes a las siguientes especies y lugar: $O$. chungarensis del Lago Chungará $(\mathrm{n}=20), O$. parinacotensis del Bofedal de Parinacota $(\mathrm{n}=28), O$. piacotensis de la laguna Piacota $(\mathrm{n}=34)$, O. laucaensis del río Lauca (Lauca $\mathrm{n}=20$, Cotacotani $\mathrm{n}=20$ y bofedal Misituni n=19) y especímenes de Orestias cf. agassii Valenciennes 1846 del bofedal de Copapujo $(\mathrm{n}=20)$ y bofedal de Chuviri ( $\mathrm{n}=16)$ (Figura 1, Tabla 1).

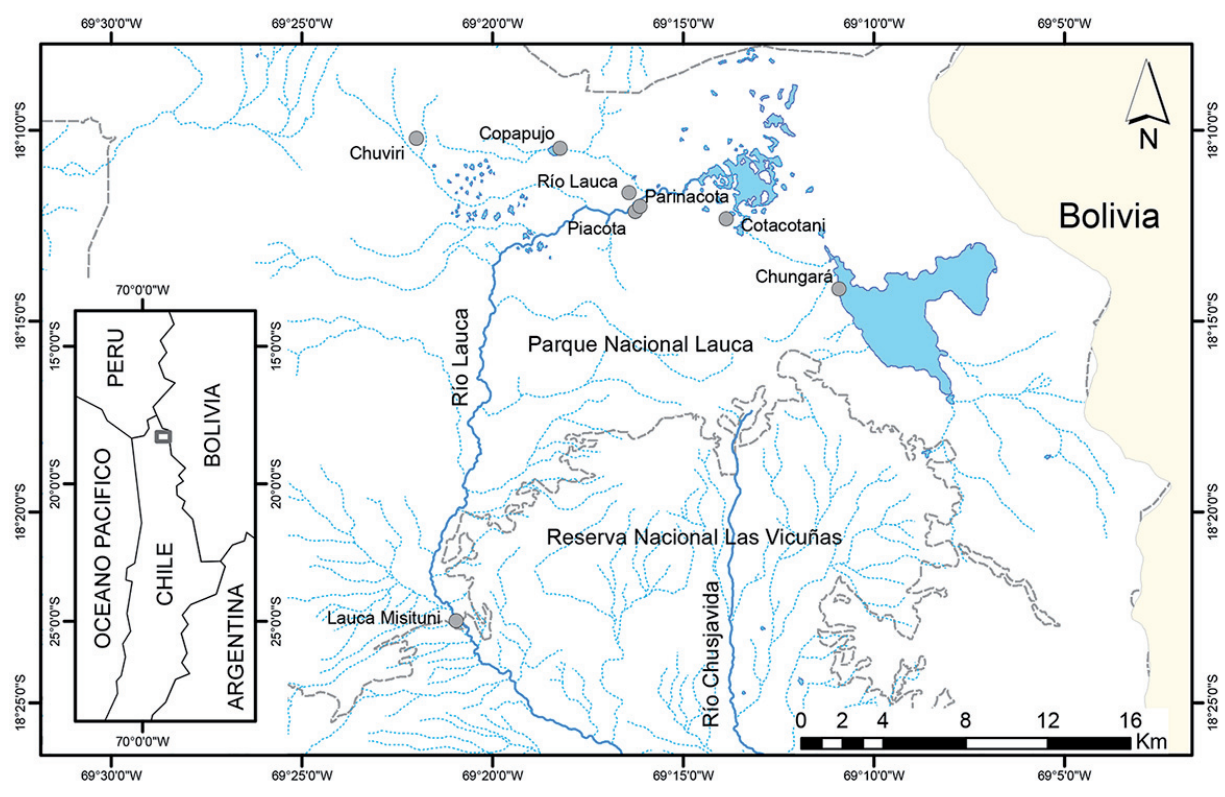

Figura 1. Mapa de Distribución de Orestias en el Parque Nacional Lauca Chile

Figure 1. Map of distribution of Orestias at Lauca National Park. Chile 
Gayana 79(1), 2015

TABla 1. Especies de Orestias en el Parque Nacional Lauca, talla estándar, ubicación geográfica de las localidades y tipo de ecosistemas .

TABLE 1. Orestias species in Lauca National Park, standard length, geographical coordinates of localities and type of ecosystems.

\begin{tabular}{|c|c|c|c|c|}
\hline Especies & $\begin{array}{l}\text { Talla } \\
\text { Promedio (DS) }\end{array}$ & Localidades & Coordenadas & Tipo de Ecosistema \\
\hline Orestias piacotensis & $26.71( \pm 4.16)$ & Piacota & $\begin{array}{l}\text { S18.19984 } \\
\text { W69.26905 }\end{array}$ & Laguna \\
\hline Orestias parinacotensis & $29.26( \pm 8.18)$ & Parinacota & $\begin{array}{l}\text { S18.20200 } \\
\text { W69.27105 }\end{array}$ & Bofedal \\
\hline Orestias chungarensis & $27.63( \pm 5.24)$ & Chungará & $\begin{array}{l}\text { S18.23820 } \\
\text { W69.18296 }\end{array}$ & Lago \\
\hline Orestias laucaensis & $31.30( \pm 4.97)$ & Cotacotani & $\begin{array}{l}\text { S18.20530 } \\
\text { W69.23126 }\end{array}$ & Laguna \\
\hline Orestias laucaensis & $40.80( \pm 8.23)$ & Lauca & $\begin{array}{l}\text { S18.19379 } \\
\text { W69.27360 }\end{array}$ & Río \\
\hline Orestias laucaensis & $45.79( \pm 11.66)$ & Misituni & $\begin{array}{l}\text { S18.38074 } \\
\text { W69.34920 }\end{array}$ & Bofedal \\
\hline Orestias agassii & $21.02( \pm 6.33)$ & Chuviri & $\begin{array}{l}\text { S } 18,16925 \mathrm{~W} \\
69,33481\end{array}$ & Bofedal \\
\hline Orestias agassii & $24.68( \pm 8.85)$ & Copapujo & $\begin{array}{l}\text { S } 18,16925 \mathrm{~W} \\
69,30785\end{array}$ & Bofedal \\
\hline
\end{tabular}

Todas las capturas fueron realizadas en la estación de prelluvias desde el momento en que los peces inician su alimentación después del deshielo diario (las 11:00 y 15:00 horas) (Northcote 2000). Los especímenes fueron sometidos a eutanasia utilizando $100 \mathrm{mg} \mathrm{l}^{-1}$ tricaine methanosulfonato, preservados en etanol $95 \%$ y mantenidos en el Laboratorio de Ecología Molecular de la Universidad de Chile hasta su análisis.

\section{ANÁLISIS DE DIETA}

Los ejemplares capturados fueron medidos (longitud estándar) y eviscerados. El contenido estomacal de cada individuo fue analizado bajo lupa estereoscópica. Se identificó y cuantificó los taxa hasta el nivel de género utilizando claves taxonómicas (Dejoux \& Iltis 1991; Figueroa 2003; Molina \& Vila 2006; Oyanedel 2013).

Los contenidos estomacales fueron analizados mediante el método de frecuencia de ocurrencia (número de estómagos con presencia de cada presa), y el método numérico (composición numérica) (Hyslop 1980). Con los cálculos mencionados, se determinó el aporte relativo de cada presa en la dieta de cada especie de Orestias por localidad mediante el índice de importancia numérica (IIN) (Windell 1968)

La amplitud trófica fue analizada mediante el cálculo de la diversidad (índice de Brillouin), la dominancia ( $\mathrm{D}=1$-índice de Simpson) (Hammer et al. 2001; Magurran 2004), riqueza (número de especies en los estómagos) y abundancia relativa (proporción del número de items presas en el total de estómagos con presas) en cada especie y población de Orestias.

El tipo de dieta fue determinada mediante el cálculo de la amplitud de nicho de Levins (Bst). Este índice es independiente del número de recursos disponibles; su valor fluctúa entre 0 para un tipo de dieta generalista y 1 para un tipo de dieta especialista (Colwell \& Futuyma 1971). Adicionalmente se calculó el sobrelapamiento de la dieta entre las especies de Orestias utilizando el índice de Pianka's (1974) (Zares \& Rand 1971; Navia et al. 2007). El sobrelapamiento incrementa el índice de Pianka, y se considera biológicamente significativo si el valor excede a 0,6. (Pianka 1974). Para evaluar si el sobrelapamiento de dieta estaría determinado por los ambientes (lago, laguna, bofedal, río) se realizó un test de randomización para comparar inter e intra localidades basado en 10,000 simulaciones bajo un modelo nulo utilizando el programa Rundom Project 1.0 (Jadwiszczak 2002).

La composición dietaria fue descrita mediante un análisis de correspondencia destendenciado, todas las ordenaciones fueron realizadas con el programa $\mathrm{R}$ software package.v. 3.0 (R Core Team, 2013) (DCA. Hill \& Gauch 1980; Jongman et al. 1995; Muñoz et al. 2002; Pardo-Gandarillas et al. 2004; Ibañez et al. 2008). 


\section{RESULTADOS}

177 especímenes analizados contenían presas en su tracto digestivo. La longitud estándar de las especies de diferentes localidades varió entre los $21.02 \mathrm{~mm}$ (O.cf agassii Chuviri) a 45,79 mm (Orestias laucaensis de Misituni) (Tabla 1); la principal diferencia se observó entre las especies de O. laucaensis (Lauca y Misituni) y O. cf agassii (Chuviri y Copapujo) con $O$. parinacotensis, $O$. piacotensis y $O$. chungarensis. Sin embargo, no hay una relación directa entre la abundancia $(p=0,33)$ o riqueza $(p=0,73)$ con la longitud estándar, por lo tanto se comparó las dieta de las especies en los diferentes sitios sin corregir por la longitud estándar (Tukey test, $\mathrm{p}<0,001)$. La dieta $(\% \mathrm{~N})$ de las especies del género Orestias fue compuesta por 9 macroinvertebrados asociados con las macrófitas, junto con los componentes del zooplancton; las presas más frecuentes fueron Cladocera, (Chydorus, Daphnia y Alonella), Ostracoda (Cyprinotus), Amphipoda (Hyalella), Mollusca (Biomphalaria.) e Insecta (Chironomus) (Tabla 2). Algunas de las especies de Orestias mostraron diferencias en las presas, tal es el caso de $O$ piacotensis que se alimentó principalmente del género Chydorus $(78,49 \%)$; O chungarensis incluye a Nauplius (40,24\%); la dieta de O. laucaensis se compone principalmente de Biomphalaria (65,22\% en Cotacotani) y O. cf.agassii en Chuviri de Hyalella (54,10\%). Algunas especies de Orestias mostraron similitudes en las presas, como son las larvas y adultos de género Chironomus para O. parinacotensis $(47,25 \%)$, O. laucaensis $(20,87 \%$ en Cotacotani y $33,47 \%$ en Misituni) y Orestias cf.agassii en Chuviri (21,31\%) (Tabla 2).

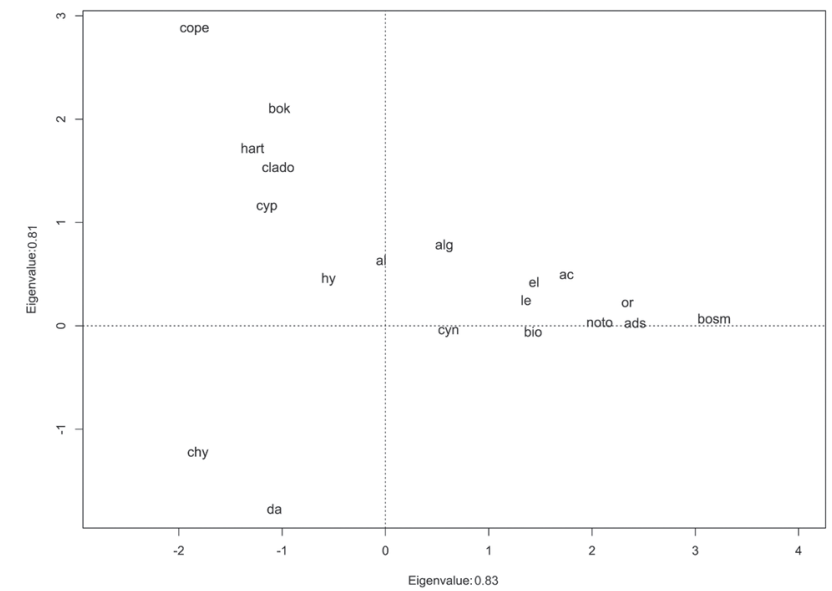

En términos de la frecuencia de ocurrencia $(\% \mathrm{~F})$ se obtuvieron diferencias entre especies, Chydorus fue la presa más importante para $O$. piacotensis $(70,59 \%)$, Chironomus para O. parinacotensis $(78,49 \%)$ y Alonella $(60 \%)$ para $O$. chungarensis. Para las localidades donde habitan $O$. laucaensis la presa más abundante fue Biomphalaria (50\% Cotacotani, 78,95\% Lauca, 68,42\% Misituni) y para Orestias cf.agassii fue Hyalella $(56,25 \%$ Chuviri and $60 \%$ Copapujo) (Tabla 2). La riqueza promedio varió entre cinco y siete especies presas para todas las especies de Orestias a excepción de O.chungarensis que mostró la mayor riqueza (13). En términos de la abundancia relativa Orestias cf agassii de Copapujo mostró el mayor número de presas por estómago $(40,75)$, seguido de $O$. chungarensis $(28,95)$ y $O$. piacotensis $(21,88)$. Por el contrario, Orestias cf. agassii de Chuviri mostró la abundancia relativa más baja $(3,81)$ (Tabla 3).

La diversidad en la dieta fue más variable: $O$. chungarensis presentó la mayor diversidad $(1,72)$, mientras $O$. piacotensis mostró la diversidad más baja $(0,78)$ (Tabla 3$)$. Los valores de amplitud de dieta indicaron valores intermedios entre las estrategias especialista y generalista Bst $(<0.5)$ (Table 3$)$. Los análisis de sobrelapamiento de dieta mostraron valores entre 0,01 (Chungará-Lauca) y 0,72 (Parinacota-Misituni). Los valores de sobrelapamiento calculados entre individuos de diferentes sistemas acuáticos no difieren significativamente de los valores calculados por iteraciones (Random test) ( $\mathrm{p}=0.64$ ); así mismo las muestras de O. laucaensis de Cotacotani, Lauca y Misituni tampoco mostraron valores significativos de sobrelapamiento $(\mathrm{p}=0,11)$ (Tabla 4).

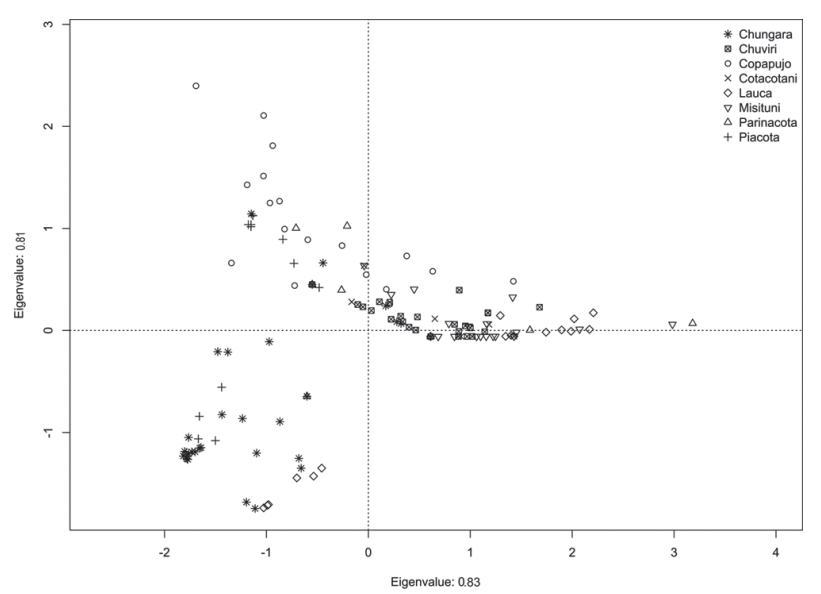

Figura 2. Bi-plot de Análisis de Correspondencia Destendenciado mostrando la posición de las presas (a)y los sitios de las especies de Orestias en un espacio reducido. Nomenclatura presas:hy: Chydorus. Da: Dapnhia. Hy: Hyallela. Cyn: Chyronomus. Bio: Biomphalaria. Cyp:Cyprinotus. Al: Alonella. Le: Leucotichia. El: Elmes. Or: Orestias egg. Ac. Acylus.Bok: Boekella. Hart: Harpacticoidea. Naupl: Nauplius. Alg: Algae. Noto: Notonecta. Dia: Diacyclops. Ads: Adesioups. Bosm. Bosmina.

Figure 2. Bi-plot ordination of Detrended Correspondence Analysis showing the position of prey (a) and Orestias species (b) in a reduced space. Nomenclature prey: Chy: Chydorus. Da: Dapnhia. Hy: Hyallela. Cyn: Chyronomus. Bio: Biomphalaria. Cyp:Cyprinotus. Al: Alonella. Le: Leucotichia. El: Elmes. Or: Orestias egg. Ac. Acylus.Bok: Boekella. Hart: Harpacticoidea. Naupl: Nauplius. Alg: Algae. Noto: Notonecta. Dia: Diacyclops. Ads: Adesioups. Bosm. Bosmina. 


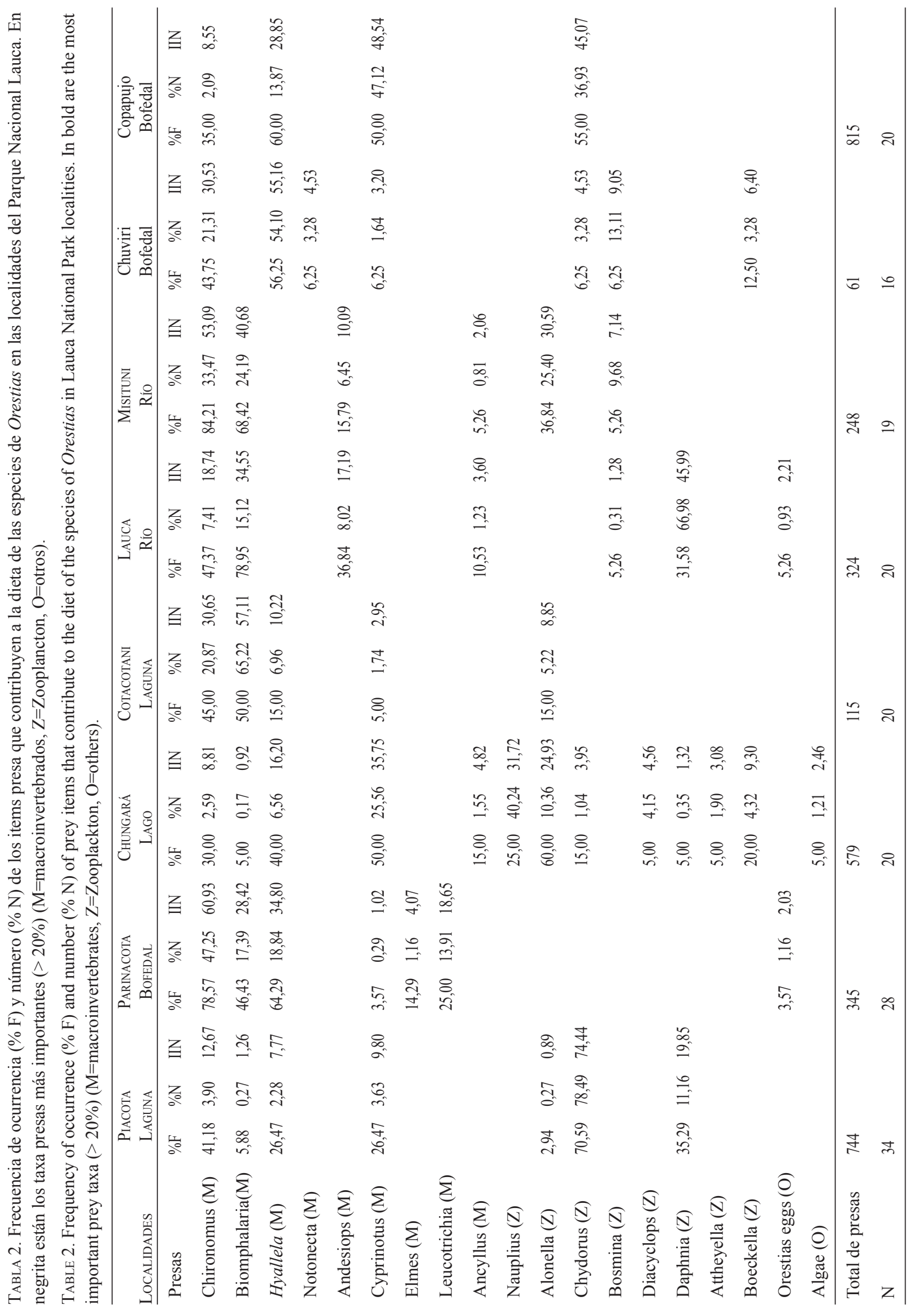


Los cuatro primeros eigenvalues del DCA de los 19 items presas y los 177 individuos de Orestias para las ocho localidades fueron $1_{1}=0,85,1_{2}=0,81,1_{3}=0,71,1_{4}=0,70$. Los dos primeros gradientes de composición explican el 55,1\% de la variación en la dieta. Con una asociación significativa entre predador y las presas $\left(\mathrm{c}^{2}{ }_{119}=7874,7, \mathrm{p}<0,001\right)$, que indica que los individuos de las diferentes poblaciones de Orestias presentan presas similares con solo diferencias en su abundancia. En los gráficos dimensionales algunas presas están localizadas en los extremos de los ejes del gradiente de composición (Figura 2 a). La composición de la dieta de Orestias de las ocho localidades mostraron asociaciones significativas entre los items presa con mayor frecuencia $(>20 \%)$ entre las localidades Parinacota, Misituni y Cotacotani; y entre las localidades Chungará, Piacota y Lauca (Figura 2 b).

TABla 3. Riqueza (Rich), abundancia relativa (A.R), amplitud de nicho (Bst),diversidad (HB), dominancia (D) en los estómagos de las diferentes especies de Orestias en el Parque Nacional Lauca.

TABLE 3. Richness (Rich), relative abundance (A.R), niche breadth (Bst), diversity (HB), Dominance (D) in stomach of different species of Orestias in Lauca National Park.

\begin{tabular}{lllllllll}
\hline & $\begin{array}{l}\text { Orestias } \\
\text { piacotensis } \\
\text { Piacota } \\
(\mathrm{n}=34)\end{array}$ & $\begin{array}{l}\text { Orestias } \\
\text { parinacotensis } \\
\text { Parinacota } \\
(\mathrm{n}=28)\end{array}$ & $\begin{array}{l}\text { Orestias } \\
\text { chungarensis } \\
\text { Chungará } \\
(\mathrm{n}=20)\end{array}$ & $\begin{array}{l}\text { Orestias } \\
\text { laucaensis } \\
\text { Cotacotani } \\
(\mathrm{n}=20)\end{array}$ & $\begin{array}{l}\text { Orestias } \\
\text { laucaensis } \\
\text { Lauca } \\
(\mathrm{n}=20)\end{array}$ & $\begin{array}{l}\text { Orestias } \\
\text { laucaensis } \\
\text { Misituni } \\
(\mathrm{n}=19)\end{array}$ & $\begin{array}{l}\text { Orestias } \\
\text { cf. agassii } \\
\text { Chuviri } \\
(\mathrm{n}=16)\end{array}$ & $\begin{array}{l}\text { Orestias } \\
\text { cf. agassii } \\
\text { Copapujo } \\
(\mathrm{n}=20)\end{array}$ \\
\hline Rich & 7 & 7 & 13 & 5 & 7 & 6 & 5 & 6 \\
A.R & 21,88 & 12,32 & 28,95 & 5,75 & 17,05 & 13,05 & 3,81 & 40,75 \\
Bst & 0,10 & 0,37 & 0,25 & 0,27 & 0,18 & 0,60 & 0,45 & 0,33 \\
HB & 0,78 & 1,33 & 1,72 & 0,95 & 1,03 & 1,45 & 1,19 & 1,07 \\
D & 0,63 & 0,31 & 0,25 & 0,48 & 0,48 & 0,25 & 0,36 & 0,38 \\
\hline
\end{tabular}

TABla 4. Sobrelapamiento de dieta de Orestias en el Parque Nacional Lauca.

TABle 4. Diet overlap of Orestias in Lauca National Park.

\begin{tabular}{|c|c|c|c|c|c|c|c|}
\hline & $\begin{array}{c}\text { PIACOTA } \\
\text { o. piacotensis }\end{array}$ & $\begin{array}{c}\text { PARINACOTA } \\
\text { o. parinacotensis }\end{array}$ & $\begin{array}{c}\text { CHUNGARÁ } \\
\text { o. chungarensis }\end{array}$ & $\begin{array}{c}\text { СотAсотANI } \\
\text { o. laucaensis }\end{array}$ & $\begin{array}{c}\text { LAUCA } \\
\text { o. laucaensis }\end{array}$ & $\begin{array}{c}\text { MisitUNI } \\
\text { O.laucaensis }\end{array}$ & $\begin{array}{c}\text { CHUVIRI } \\
\text { o. } \text { cf agassii }\end{array}$ \\
\hline Piacota & - & & & & & & \\
\hline Parinacota & 0,053 & - & & & & & \\
\hline Chungará & 0,052 & 0,093 & - & & & & \\
\hline Cotacotani & 0,022 & 0,587 & 0,061 & - & & & \\
\hline Lauca & 0,141 & 0,159 & 0,014 & 0,238 & - & & \\
\hline Misituni & 0,036 & 0,723 & 0,143 & 0,700 & 0,193 & - & \\
\hline Chuviri & 0,099 & 0,609 & 0,158 & 0,199 & 0,039 & 0,281 & - \\
\hline
\end{tabular}

\section{DISCUSIÓN}

En los procesos de especiación ecológica, el uso diferencial del recurso es un factor importante para el desarrollo de especies incipientes (Schluter 2001; Wiens 2004). En simpatría, las condiciones de competencia promueven la selección diferencial de los recursos, que es evidente en una especialización dietaria. Por el contrario, dada la ausencia de competencia interespecífica durante la especiación alopátrica, las especies incipientes podrían mantener una estrategia trófica generalista. (Elmer et al. 2010; Scharnweber et al. 2011). En los sistemas acuáticos en climas extremos, como es el caso del Altiplano, donde la especiación ha estado ocurriendo en los últimos 12 mil años, es importante conocer la respuesta alimentaria de las especies que habitan estos sistemas (Giralt et al. 2008; Vila et al. 2013). Localizados sobre los 4000 msnm, ellos se caracterizan por ser de baja profundidad, sin interconexiones, con características físicas y químicas particulares y un cinturón de macrófitas y biodiversidad similares (Guzmán \& Sepulveda 2009, Marquez et al. 2009; Riveros et al. 2012, Oyanedel 2013).

Acorde con la información histórica del Parque Nacional Lauca, los ambientes acuáticos donde habitan Orestias presentan características extremas como son los altos valores de radiación solar, oscilaciones drásticas de temperatura diaria y baja variación estacional.(Risarcher 
et al 2003) Estas condiciones tienden a producir una baja biodiversidad asociada con el cinturón de macrófitas en los cuales las especies de Orestias habitan (Guerrero 2013; Oyanedel 2013). En el PNL, las cinco especies de Orestias analizadas mostraron semejanzas en las riquezas de presas por sitio (entre cinco y siete), con la diferencia de $O$. chungarensis que presentó una mayor riqueza (Riqueza $=$ 13). La abundancia de presas varió dependiendo del sistema acuático, por ejemplo Hyalella se encontró con mayor abundancia en los bofedales, de manera similar ocurrió con Chironomus y Biompahalaria, mientras Chydorus es más una presa lacustre. Esta baja riqueza estaría asociada con la baja amplitud de dieta y la baja diversidad de presas que puede ser explicada por el tamaño reducido y la baja profundidad de los sistemas, los cuales tienen pocos taxa asociados en los cinturones de vegetación de macrófitas (Dorador et al. 2003; Riveros et al. 2012). Por otro lado la amplitud dietaria indicó valores límites entre un tipo de dieta especialista y generalista. Adicionalmente, los valores de sobrelapamiento de nicho no fueron biológicamente significativos entre las cinco especies en los diferentes ambientes $(\mathrm{p}>0.5)$. En el caso del Parque Nacional Lauca, donde cada especie habita un sistema acuático, una amplitud de dieta reducida y un sobrelapamiento no significativo podría está ligado a la similitud de macroinvertebrados y zooplancton que tienen los ambientes, que sólo varían en la abundancia (Marquez et al. 2009; Oyanedel 2013). Así mismo, no hubo diferencias significativas en el sobrelapamiento de presas entre ambientes (lago, laguna, bofedal, río), por lo tanto las diferencias entre dietas no estarían asociadas en función del hábitat.

La variación en la riqueza de presa, la amplitud de dieta y el sobrelapamiento observado entre las poblaciones de Orestias podrían reflejar un tipo de dieta oportunista, consumiendo las especies presas con mayor frecuencia en los ambientes. Estas diferencias en la dieta entre las poblaciones locales de Orestias podrían estar asociadas a la variación de la disponibilidad de las presas, especialmente en situaciones de condiciones climáticas extremas como es el Parque Nacional Lauca (Habets et al. 2006).

Debido a que la zona de estudio se encuentra sometida a la extracción de agua e introducción de especies, este estudio entrega información importante en relación a la alimentación de Orestias para el manejo de la extracción y explotación de los recursos hídricos en el río Lauca y los sistemas acuáticos asociados a este.

\section{AGRADECIMIENTOS}

Agradecimientos al Dr. Christian Ibáñez por la ayuda en los análisis de datos, a Michel Sallaberry y Sergio Scott por la ayuda en la colecta de los especímenes. Este estudio fue financiado por los proyectos Fondecyt 1110243 y 1140543. Proyecto P05-002 ICM. Financiado ICM - MINECON, P05-002 IEB. Al Instituto de Ecología y Biodiversidad, y a la beca de estudios doctorales en Chile 2007-2011 (D20107004) de Comisión Nacional Científica y Tecnológica Conicyt.

\section{BIBLIOGRAFIA}

Arratia, G. 1982. Peces del Altiplano de Chile. En: El Hombre y los Ecosistemas de Montaña (Eds. Veloso, A. \& E., Bustos). pp. 93-133. Oficina Regional de Ciencia y Tecnología de la UNESCO para América Latina y el Caribe, Montevideo, Uruguay.

Clavero, J.E., Sparks, S. J., Polanco, E. \& Pringle, M. 2004. Evolution of Parinacota volcano, Central Andes, Northern Chile. Revista Geológica de Chile 31:317-347.

Colwell, R. \& Futuyma, D. J. 1971. On the measurement of niche breadth and overlap. Ecology 52:567-572.

Costa, W.J. 2003. Family Cyprinodontidae (Pupfishes). In: Check List of the Freshwater Fishes of South and Central America (Eds. Reis, R.E, Kullander, S.O. \& Ferraris, C.J.), pp. 549554. Edipucrs. Porto Alegre. Brazil.

Dejoux, C. \& Iltis, A.1991. El Lago Titicaca. Síntesis del conocimiento limnológico actual. ORSTOM. HISBOL, La Paz, Bolivia. 584 pp.

Dorador, C., Pardo R. \& Vila, I. 2003.Variaciones temporales de parámetros físicos, químicos y biológicos de un lago de altura: el caso del lago Chungará. Revista Chilena de Historia Natural 76:15-22.

Elmer, K. R., Lehtonen, T.K., Kautt, A.F., Harrod, C. \& Meyer, A. 2010. Rapid sympatric ecological differentiation of crater lake cichlid fishes within historic times. BioMed Central Biology 8:60.

Figueroa, R., Valdovinos, C., Araya, E. \& Parra, O. 2003. Macroinvertebrados bentónicos como indicadores de calidad del agua de ríos del sur de Chile. Revista Chilena de Historia Natural 76:275-285.

Giralt, S., Moreno, A., Bao, R., SÁez, A., Prego, R., ValeroGarcés, B., Pueyo, J., GonzÁlez-SAmpériz, P. \& Taberner, C. 2008. A statistical approach to disentangle environmental forcings in a lacustrine record: the Lago Chungará case (Chilean Altiplano). Journal of Paleolimnology 40(1): 195-215.

Guerrero, C.J. 2013. Diferenciación genética y ecológica en el complejo agassizii del género Orestias en las cuencas de la Puna del noreste de Chile. PhD Thesis. Facultad de Ciencias, Universidad de Chile. Santiago. Chile.

Habets, M., Rozen, D., Hoekstra, R. \& de Visser, J. 2006. The effect of population structure on the adaptative radiation of microbial populations evolving in spatially structured environments. Ecology Letters 9:1041-1048.

Hammer, Ø., Harper, D.A.T. \& Ryan, P.D. 2001. PAST: Paleontological Statistics Software Package for Education and Data Analysis. Palaeontologia Electronica 4(1): 9pp.

Hill, M.O. \& Gauch, H.G. 1980. Detrended correspondence analysis: an improved ordination technique. Vegetatio 42:47-58. 
HysLop, J. 1980. Stomach contents analysis: a review of methods and their application. Journal of Fish Biology 17:411-429.

Ibañez, C.M., Arancibia, H., \& Cubillos, L.A. 2008. Biases in determining the diet of jumbo squid Dosidicus gigas (D' Orbigny 1835) (Cephalopoda: Ommastrephidae) off southern-central Chile $\left(34^{\circ} \mathrm{S} \_40^{\circ} \mathrm{S}\right)$. Helgoland Marine Research 62:331-338.

JADwiszczaK, P. 2002. Rundom Project 1.0. Statistical Analysis Software.

Kocher, T.D. 2004. Adaptive evolution and explosive speciation: the cichlid fish model. Nature Review Genetics 5:288-298.

MagurRan, A. 2004. Measuring biological diversity. Blackwell Science Ltd. USA. 256 pp.

Maldonado, E., Hubert, N., Sagnesk, P. \& De Mérona, B. 2009. Morphology-diet relationships in four killifishes (Teleostei, Cyprinodontidae, Orestias) from Lake Titicaca. Journal of Fish Biology 74:502-520.

Márquez-García, M., Vila, I., Hinojosa, L.F., Méndez, M.A., Carvajal, J.L. \& Sabando, M.C. 2009. Distribution and seasonal fluctuations in the aquatic biodiversity of the southern Altiplano. Limnologica 39:314-318.

Molina, X. \& Vila, I. 2006. Manual de evaluación de la calidad del agua. CENMA. Universidad de Chile. 93 pp.

Muñoz, G., Valdebenito, V. \& George-Nascimento, M. 2002. La dieta y la fauna de parásitos metazoos del torito Bovichthys chilensis Regan 1914 (Pisces: Bovichthydae) en la costa de Chile centro-sur: variaciones geográficas y ontogenéticas. Revista Chilena de Historia Natural 75:66-671.

Navia, A.F., Mejia-Falla, P.A. \& Giraldo, A. 2007. Feeding ecology of elasmobranch fishes in coastal waters of the Colombian Eastern Tropical Pacific. BioMed Central Ecology 7:8.

Northсоте, G.G. 2000. Ecological interactions among an Orestiid (Pisces: Cyprinodontidae) species flock in the littoral zone of Lake Titicaca. In: Advances in Ecological research: Ancient lakes Biodiversity, Ecology and Evolution (Eds. A. Rossiter \& H. Kawanabe), pp. 339-120. Academic Press, N. York.

Oyanedel, J.P. 2013. Topología trófica y la sensibilidad a la pérdida de especies en lagos altiplánicos. Tesis de Magister. Facultad de Ciencias, Universidad de Chile, Santiago, Chile.

Parenti, L.1984a. Biogeography of the Andean killifish genus Orestias with comments on the species flock concept, In: Evolution of Fish Species Flocks (Eds. Echelle, A.A. \& Kornfield, I.), pp. 85-92. University of Maine Press, Orono, Maine.

PARenti, L. 1984b. A taxonomic revision of the Andean killifish genus Orestias (Cyprinodontiformes, Cyprinodontidae. Bulletin of the American Museum of Natural History 178:107-214.

Pardo-Gandarillas, M.C., Garcías, F. \& George-Nascimento, M. 2004. La dieta y la fauna de endoparásitos del pejesapo Gobiesox marmoratus Jenyns, 1842 (Pisces: Gobiesocidae) en el litoral central de Chile están conectadas pero no correlacionadas. Revista Chilena de Historia Natural. 77:627-637.

PIANKA, E.R. 1974. Niche overlap and diffuse competition. Proceedings of the National Academy of Sciences of the United States. 71:2141-2145.

Risarcher, F., Alonso, H. \& Salazar, C. 2003.The origin of brines and salt in salars: a hydrogeochemical review. Earth Science Reviews 63:249-293.

R. Core. Team. 2013. R: A language and environment for statistical computing. R Foundation for Statistical Computing, Vienna, Austria. URL http://www.R-project. org/. Accesado octubre 2012.

Riveros, J., MÉndez, M. \& Vila, I. 2012. Trophic niche of Orestias agassii (Cuvier \& Valenciennes, 1846) in the streams system of Salar de Huasco (200' S; $\left.68^{\circ} 15^{\prime} \mathrm{W}\right)$. Gayana 76(2):79-91.

Sáez, A., Valero-Garcés B., Moreno, A., Bao, R., Pueyo, J., González-Sampériz, P., Giralt, S., Taberner, C., Herrera, C. \& Gibert, R. 2007. Lacustrine sedimentation in active volcanic settings: the Late Quaternary depositional evolution of Lake Chungará (northern Chile). Sedimentology 54:1191-1222.

SChluter, D. 2001. Ecology and the origin of species. Trends in Ecology and Evolution 16:372-380.

Scharnweber, K., Plath, M., Winemiller, K.O. \& Tobler, M. 2011. Dietary niche overlap in sympatric asexual and sexual live bearing fishes Poecilia spp. Journal of Fish Biology 79:1760-1773.

Vila, I. \& Pinto, M. 1986. A new species of killifish (Pisces: Cyprinodontidae) from the chilean altiplano. Revue d'Hydrobiologie Tropicale. 19 (3/4): 233-239.

VILA, I. 2006. A new species of Killifish in the Genus Orestias (Teleostei; Cyprinodontidae) from the Southern High Andes, Chile. Copeia 3:471-476.

Vila, I., Scott, S., Méndez, M., Valenzuela, F., Iturra, P. \& Poulin, E. 2011. A new species of Orestias (Teleostei: Cyprinodontidae) from southern high Andes salt pan springs. Ichthyological Exploration of Freshwaters 22:345-353.

Vila, I., Morales, P., Scott, S., Poulin, E., Véliz, D., Harrod, C. \& MÉndez, M.A. 2013. Phylogenetic and phylogeographic analysis of the genus Orestias (Teleostei: Cyprinodontidae) in the southern Chilean Altiplano: the relevance of ancient and recent divergence processes in speciation. Journal of Fish Biology 82:927-943.

WIENS, J.J. 2004. Speciation and ecology revisited: phylogenetic niche conservatism and the origin of species. Evolution 58(1):193-197.

Windell, J.T. 1968. Food analysis and rate of digestion. En: Methods for assessment of fi sh production in freshwaters (Eds. W.E. Ricker), pp. 197-203. Ed. IBP Handbook No 3, Blackwell Scientific Publ.Oxford, England.

Zares, T.M. \& Rand, A.S. 1971. Competition in tropical stream fishes: support for the competitive exclusion principle. Ecology 52:336-342. 